\title{
Heute muss man die Deutschen von Europa überzeugen
}

Ein Gespräch mit Jacques Delors

\author{
Jacques Delors $\cdot$ Sabine von Oppeln $\cdot$ Reinhard Blomert
}

von Oppeln: Welche Erwartungen und Zielvorstellungen haben Sie an die europäische Politik geknüpft, als Sie 1984 das Amt des Kommissionspräsidenten akzeptiert hatten?

Delors: Ich habe mich schon in den 1950er Jahren für Europa stark gemacht. Ich hatte immer große Bewunderung für alle, die zur Aussöhnung beigetragen und die Vision von einer europäischen Zukunft ermöglicht haben. 1984 glaubte ich, dass meine Aufgabe in Frankreich beendet sei, nachdem ich die so genannte ,politique de la rigueur" (Austeritätspolitik) eingeführt hatte - ein Wort, das man heute nicht gern in den Mund nimmt. Ich war damals auch Bürgermeister von Clichy bei Paris, einer Stadt mit 50.000 Einwohnern. Ich gab dem französischen Staatspräsidenten zu verstehen, dass ich zurück an die Universität wollte. Ich sagte ihm, meine Aufgabe sei beendet. Wie der Zufall so will, zeigte das Glücksrad genau in diesem Moment auf mich. Als überzeugter Anhänger der europäischen Idee war ich darüber natürlich sehr erfreut. Denn trotz aller Anstrengungen beim Europäischen Rat in Fontainebleau insbesondere hinsichtlich des Abkommens über die britischen Ausgleichszahlungen (dem so genannten „Britenrabatt“) musste ich miterleben, wie die Europäische Gemeinschaft - die damals aus neun bzw. zehn Mitglieds-

(c) VS-Verlag 2010

J. Delors

ehemaliger EU-Präsident

„Notre Europe“, 19, rue de Milan,

75009 Paris, Frankreich

Dr. S. von Oppeln $(\bowtie)$

Fachbereich Politik- und Sozialwissenschaften, Arbeitsstelle Europäische Integration,

FU Berlin,

Inhnestraße 22,

14195 Berlin, Deutschland

E-Mail: oppeln@zedat.fu-berlin.de

Dr. habil. R. Blomert

WZB, Reichpietschufer 50,

10785 Berlin,

Deutschland

E-Mail: blomert@wzb.eu 
staaten bestand - seit vier oder fünf Jahren schon von Zweifeln heimgesucht wurde und unter der weltweiten wirtschaftlichen Stagnation und der steigenden Arbeitslosigkeit litt.

Da sagte ich mir, dass Europa statt Diskussionen über große europäische Ideen, Föderation, Konföderation usw. vielleicht eher neue Impulse bräuchte, um die Wachstumsdynamik wieder anzukurbeln. Ich habe dieses Amt angenommen und hatte die Idee als Ansporn und zur Überwindung dieser Krise - die Konjunkturlage war ganz besonders schwierig - die Einrichtung eines europäischen Binnenmarkts vorzuschlagen. Als designierter Kommissionspräsident habe ich in diesen Monaten alle neun Hauptstädte bereist. Als dann die Kommissare ernannt wurden, habe ich es so eingerichtet, dass es keine Nacht der langen Messer gab und die Verteilung der Zuständigkeiten unter uns und ohne Einmischung der Regierungen ausgehandelt wurde. Ende November waren alle Ämter verteilt. Die Kommission konnte ihre Arbeit Anfang Januar 1985 aufnehmen.

von Oppeln: Vor Ihrer Tätigkeit als Kommissionspräsident haben Sie in Frankreich als Finanzminister die „,politique de la rigueur" eingeführt und damit der keynesianischen Politik ein Ende gesetzt. Das war eine politische Umorientierung...

Delors: Das war nicht mal eine keynesianische, sondern eine durch den Regierungswechsel bedingte Politik. Das ist kein Einzelfall in Europa. Sie hatten in Deutschland ja auch christdemokratisch-liberale, sozialdemokratische Regierungen und bis vor kurzem noch eine Große Koalition. Ein Regierungswechsel hat seinen Preis. Frankreichs Rechte war seit 1958 an der Macht, und da können Sie sich vorstellen, welche großen Hoffnungen die andere Seite mit einem politischen Machtwechsel verband. Präsident Mitterrand blieb gar nichts anderes übrig, als einige dieser Forderungen und Wählererwartungen zu erfüllen - und dies noch dazu in einem wirtschaftlich sehr ungünstigen Umfeld, d. h. der Dollarkurs stieg und wir hatten eine weltweite Rezession. Erschwerend kam die Tatsache hinzu, dass diese Regierung nicht das Vertrauen der anderen Staatsoberhäupter Europas hatte. Ich erinnere mich noch an den ersten Besuch von Helmut Schmidt bei François Mitterrand in seinem Landhaus im Südwesten Frankreichs. Ich kannte Helmut Schmidt gut, er hatte eher Vertrauen zu mir. Er wirkte jedoch etwas zögerlich, verunsichert und fragte sich wohl, wie das alles weitergehen sollte. Jeder Machtwechsel hat eben seinen Preis, das darf man nie vergessen, es geht eben nicht nur um hochfliegende Ziele. So hatte ich die undankbare Rolle, ständig daran erinnern zu müssen, dass aufgrund unseres steigenden Handelsdefizits und unserer schwachen Währung manche Dinge nicht möglich sind, bis zu jenem Tag, als ich mit der ,ppolitique de la rigueur“ einen Kurswechsel einleiten konnte - ein Begriff, der übrigens bis heute noch nicht akzeptiert ist. Doch dieser Kurswechsel war unumgänglich. Die Regierungen haben sich bei ihrer Wahl für den Kommissionspräsidenten wahrscheinlich auch deswegen für mich entschieden, weil sie wussten, dass ich außerhalb und innerhalb eines Landes streng auf das Budget achte.

von Oppeln: Doch vielleicht erhoffte sich die französische Politik damals auf europäischer Ebene eine Art Kompensation für diesen Wandel der Politik auf nationaler Ebene zu finden? 
Delors: Nein, ich betone, dass - wenn man das deutschen Lesern sagen kann, was mir wünschenswert oder zumindest nützlich erscheint - François Mitterrand ein großer Europäer war. Er nahm 1948 am Haager Kongress unter dem Vorsitz Winston Churchills teil, der gewissermaßen den Anstoß gab, und unsere deutschen Freunde waren auch dabei. Mitterrand verstand sich also immer als Europäer. Nur war es schwierig für ihn, sein europäisches Ideal mit den Belangen seines eigenen Landes in Einklang zu bringen; das war nicht leicht. Dennoch war er seit Anbeginn Europäer und unterbreitete trotz meiner gelegentlichen Warnungen Vorschläge zu Europa, u. a. zur Industriepolitik. Seine Partner hatten natürlich mehr oder weniger Vorbehalte bzw. waren mehr oder weniger kritisch gegenüber seiner Innenpolitik. Doch die wichtige Entscheidung für die Austeritätspolitik im Jahr 1983 war größtenteils seinem europäischen Engagement geschuldet. Ich hatte ihm geraten, nicht das europäische Währungssystem zu verlassen, das auf Initiative von Helmut Schmidt und Valéry Giscard D'Estaing ins Leben gerufen worden war. Das ist die ehrliche Erklärung für diese Situation. Ich verheimliche Ihnen nichts, ich sage Ihnen die Dinge genau so, wie ich sie sehe.

Blomert: Von wem stammte denn die Idee eines europäischen Binnenmarkts und der Währungsunion? War das schon ein alter Plan gegen den imperialistischen Dollar?

Delors: Ich verstehe, was Sie meinen. Man muss zwei Dinge unterscheiden. Als ich zum Kommissionspräsidenten ernannt wurde, war es mein Ziel, Europa zu motivieren und den Europäern wieder Selbstvertrauen zu geben. Hinzu kam, dass zwischen den Ländern so große Uneinigkeit herrschte, dass manche meiner späteren Vorschläge hinsichtlich des Binnenmarkts und der Zielvorgabe 1992, die bereits von der vorigen Kommission unter Präsident Thorn eingebracht worden waren, nicht beschlossen werden konnten, denn damals galt noch das Prinzip der Einstimmigkeit. Als sie also meine Zielvorgabe 1992 akzeptierten, sagte ich ihnen, entweder ändert Ihr den Vertrag oder Ihr enthaltet Euch, wenn Ihr nicht einverstanden seid. Und so haben sie für die Änderung des Vertragswerks gestimmt. Der entscheidende Punkt war die Verabschiedung des Vertragsentwurfs der Einheitlichen Europäischen Akte im Jahr 1986. Nun konnte über alle Belange des europäischen Binnenmarkts, der von allen akzeptiert wurde, mit Mehrheitsentscheidungen beschlossen werden. Bei meiner Reise durch die Hauptstädte hatte ich ihnen das natürlich nicht angeboten, das war meine Reservekarte. Ich hatte ihnen eine Währungsunion, Änderungen im institutionellen Bereich und eine gemeinsame Verteidigung vorgeschlagen, doch die zehn Staaten waren sich uneins. Der einzige Punkt, in dem sie sich einig waren, war der europäische Binnenmarkt: „Wenn Sie meinen, dass wir damit unseren gemeinsamen Markt ankurbeln können ..." So ist dieser Binnenmarkt entstanden.

Blomert: Doch dieser europäische Binnenmarkt war offen und war nicht das, was die Amerikaner befürchteten, eine Art von Festung...

Delors: Damals waren die Amerikaner sehr skeptisch im Hinblick auf den zukünftigen Aufbau Europas. Die Tatsache, dass sich die Europäer selbst zu einem ehrgeizigen Pro- 
gramm verpflichtet hatten, hat sie ein wenig beruhigt, aber nicht sehr viel. Während meiner Zeit in der EU-Kommission flog ich drei-, viermal pro Jahr in die USA und stieß dort auf gewaltige Skepsis ...

Die Folge davon jedenfalls war, dass die Einheitliche Europäische Akte selbstverständlich auch Bestimmungen über Umweltpolitik und über die Solidarität zwischen den Regionen enthielt. Beispielsweise belief sich der Fonds für wirtschaftlich schwache und rückständige Regionen bei meinem Amtsantritt auf 5 Milliarden Euro und ist heute auf 40 Milliarden Euro aufgestockt worden. Und sie enthielt auch soziale Bestimmungen und sogar einen kleinen Verweis auf die europäische Währungseinheit ECU, die übrigens damals allerdings nur widerstrebend akzeptiert wurde, auch von den Deutschen - aber diese Zeiten sind vorbei. Das war wie dieses Märchen vom kleinen Däumling, der Kieselsteine auf dem Weg fallen ließ, damit die sieben Brüder den Weg zurück wieder finden konnten. So habe auch ich den Weg für die Zukunft mit kleinen Steinen ausgelegt. Aber ich habe die Regierungschefs nicht überfordert, ich wollte nur wissen, ob sie einen europäischen Binnenmarkt haben wollten, wozu sie sich 1957 mit dem EWG-Vertrag über die Schaffung eines gemeinsamen Marktes ja verpflichtet hatten. Jedenfalls kurbelte der Binnenmarkt die europäische Wirtschaft an, 210 Gesetzestexte wurden innerhalb von drei Jahren verabschiedet, und durch das Wirtschaftswachstum und die sinkende Arbeitslosigkeit waren die Europäer so optimistisch gestimmt, dass ich gar keine Währungsunion mehr vorzuschlagen brauchte. Diese Rolle übernahmen Genscher und Balladur, allerdings in unterschiedlichen Formen.

von Oppeln: Sie sind einerseits ein Gründervater des Binnenmarkts und Initiator der Wirtschafts- und Währungsunion, haben aber andererseits stets betont, dass Sie ein Europa ablehnen, das nur auf den Markt bzw. auf eine Freihandelszone ohne Seele, ohne Gewissen, ohne politischen Willen und ohne soziale Dimension reduziert ist. Was bedeutet für Sie die soziale Dimension Europas? Sie haben ja bereits erwähnt, dass es dazu einige Passagen in dem Vertrag gibt ...

Delors: Die Trias, die ich zu jener Zeit propagierte, beinhaltete den Wettbewerb, der die Wirtschaftsentwicklung stimulieren sollte und den wir unbedingt brauchten, dann die Kooperation...

Blomert: Zwischen den Mitgliedsstaaten oder nach außen?

Delors: Zwischen den Mitgliedern und nach außen gerichtet. Es handelte sich nicht um das Konzept einer Festung Europa. Die Kooperation lag mir damals sehr am Herzen. Wir kommen darauf vielleicht noch zurück, das ist die heutige Schwachstelle der Union. Und schließlich die Solidarität, die verbindet. Solidarität in den Bereichen, in denen sie durch die Verträge ermöglicht wird. Im Bereich der Strukturfonds für wirtschaftlich schwache oder rückständige Regionen oder auch in Fragen des Budgets funktioniert das sehr gut. Im Hinblick auf die soziale Dimension hatte ich lediglich zur Bedingung gemacht, dass im Vertrag die Bedeutung des sozialen Dialogs zwischen den Arbeitgebern und den Gewerkschaften oder zwischen den Arbeitgebern, den Gewerkschaften und den öffentlichen Institutionen aufgenommen wird. Noch vor meinem Amtsantritt 
als Kommissionspräsident habe ich mich dieser Herausforderung gestellt und im Januar 1985 ein Treffen mit den Gewerkschaften und den Arbeitgebern anberaumt. Das war mit sehr viel Arbeit verbunden, denn die Gewerkschaften waren untereinander nicht immer einig, die Arbeitgeber genauso wenig. Ich habe auch eine neue Richtlinie zur Verbesserung der Sicherheit und des Gesundheitsschutzes am Arbeitsplatz initiiert. Doch darüber hinaus hat der Vertrag im Bereich der Sozialpolitik viele Kompetenzen bei den Mitgliedsstaaten belassen.

Ich war niemals darauf aus, dies zu ändern. Das wäre unrealistisch gewesen. Zwischen den Wohlfahrtsstaaten in Deutschland, Großbritannien, Frankreich und den nordischen Staaten gibt es beträchtliche Unterschiede. Die soziale Dimension europäischer Politik beinhaltete für mich in erster Linie die Solidarität innerhalb der Europäischen Union und schließlich den sozialen Dialog mit seinen Fortentwicklungen. Wir haben am 30. November in Brüssel, nicht in Frankreich - denn wir können von Frankreich nicht mehr verlangen als es zu geben imstande ist -, das 20-jährige Bestehen der Sozialcharta über die Grundrechte der Arbeitnehmer gefeiert, die im Dezember 1989 vom Europarat mit einem einzigen ,opting-out' von Großbritannien, natürlich unter Margaret Thatcher, ratifiziert worden war. Ausgehend von dieser Charta haben wir uns auf einen Minimalkonsens geeinigt. Keine Harmonisierung nach oben, aber immerhin ein Mindestprogramm. Jahrelang habe ich dafür gekämpft, aber niemals habe ich die Verlagerung der wohlfahrtsstaatlichen Politik in den europäischen Zuständigkeitsbereich gefordert. Das war nicht realistisch. Das führte zu einem großen Missverständnis mit den Franzosen, die gehofft hatten, Europa würde die Probleme lösen, die Frankreich bei sich nicht hatte lösen können. Dieser Irrtum erklärt auch das ,Nein‘ Frankreichs zum EU-Referendum 2005.

von Oppeln: Und wie sehen Sie heute die Entwicklung der sozialen Dimension und auch des sozialen Dialogs auf europäischer Ebene? Sind Sie eher zufrieden oder unzufrieden?

Delors: Ich möchte niemandem zu nahe treten, aber es funktioniert nicht mehr. Das Herz ist nicht mehr dabei, es gibt keinen Elan mehr. Wenn Sie so wollen, was hat die Globalisierung neben ihren positiven Aspekten gebracht? Erstens hat sie praktisch einen weltweiten Arbeitsmarkt geschaffen, was die europäischen Arbeitnehmer natürlich zu spüren bekommen. Das muss man akzeptieren, denn es handelt sich ja um arme Schwellenländer, aber man muss auch ausreichend wirtschaftliche Aktivität und Arbeitsplätze in Europa schaffen. Zweitens spielt der individualistische Zeitgeist eine nicht unbeträchtliche Rolle bei der Abkehr von kollektiven Organisationen wie Gewerkschaften. Zu meiner Zeit war das noch anders, ich hatte mehr Glück. Die Gewerkschaften waren am Ziel Europa, am Binnenmarkt interessiert und daher zu Konzessionen bereit. Sie haben nicht alles rundweg abgelehnt mit dem Argument, das sei alles nur Kapitalismus und Liberalismus. Nein, sie haben das akzeptiert. Doch heute sind die Gewerkschaften in einer schwachen Position gegenüber den Arbeitgebern. Wäre ich heute Kommissionspräsident, würde ich daher versuchen, ihnen ein wenig unter die Arme zu greifen, um das Gleichgewicht der Kräfte wieder herzustellen. Die deutsche Soziale Marktwirtschaft, das skandinavische Modell und das französische System haben eins gemein: Sie streben eine Balance zwischen der Gesellschaft und dem Individuum und einen gewissen Kompromiss zwischen Kapital und Arbeit an. 


\section{Blomert: Solidarität und Wettbewerb.}

Delors: Genau, zwischen Kapital und Arbeit und auch zwischen dem Markt und den öffentlichen Institutionen. Diese Verhältnisse sind aus dem Gleichgewicht geraten. Wenn wir uns für den sozialen Dialog einsetzen, muss im Moment also eher den Gewerkschaften geholfen werden. Man darf nicht übertreiben, aber sie brauchen Unterstützung. Denn wenn uns dieses Gleichgewicht verloren geht, dann verlieren wir die europäische Seele. Das ist dann ein anderes Modell, und zwar ein Modell, in dem sich das Individuum auf Kosten der Gesellschaft entfaltet und über sie triumphiert. Oder auch das asiatische Modell, bei dem die Gesellschaft über den Einzelnen herrscht und ihn an seiner Entfaltung hindert. Unserem Modell hingegen liegt eine andere Philosophie zugrunde. Und dieses Modell wird derzeit in all seinen Ausprägungen gefährdet. Dabei gibt es, wie gesagt, erhebliche Unterschiede zwischen dem britischen Dritten Weg und dem vorhergehenden BeveridgeModell, zwischen dem deutschen Modell und dem etwas zentralistischer geprägten französischen System und dem krisenfesteren und subtileren skandinavischen Modell. All dies wird derzeit in Frage gestellt. Da ist es nicht verwunderlich, dass die Arbeitnehmer angesichts dieser Bedingungen die Globalisierung und Europa fürchten. Das ist eine Tatsache und kein ideologisch gefärbtes Urteil, sondern eine reine Feststellung.

\section{Blomert: War die Osterweiterung vielleicht ein Fehler, weil sie die Solidarität der Gewerkschaften schwächt?}

Delors: Nein, wenn Sie so wollen, war das eine historische Herausforderung. Was würden wohl die Historiker im Jahre 2030 sagen, wenn Europa die Osterweiterung abgelehnt hätte? Sie würden Europa als ein Konstrukt aus egoistischen Ländern mit einem relativ hohen Lebensstandard betrachten, den man den anderen Ländern vorenthalten hätte, obwohl sie ebenfalls zu Europa gehören. Sie würden feststellen, dass Europa weder aus dem Berliner Mauerfall noch aus dem Untergang des Kommunismus Konsequenzen gezogen hätte. Wir konnten gar nicht anders handeln! Hätten wir es etwas besser machen können? Sicherlich, aber soweit sind wir noch nicht ... Wir haben die Wahl zwischen denen, die meinen, wir hätten alles falsch gemacht - außer in Frankreich wird das allerdings nirgendwo laut geäußert - und denen, die wie ich denken, dass wir unsere Arme öffnen mussten. Man hat dies getan, und das hat seinen Preis. Man darf nicht vergessen, dass die Wirtschaftsentwicklung in manchen Beitrittsländern zum Zeitpunkt ihres Beitritts im Jahre 2004 nur 30\% des Entwicklungsniveaus der alten Mitgliedsstaaten betrug. Das ist der Beweis dafür, dass Europa vor einer weltweiten Herausforderung an Großzügigkeit nicht zurückgeschreckt ist. Natürlich ist es rentabler, Automobilwerke in der Slowakei als in Deutschland und Frankreich anzusiedeln. Doch konnten wir diese historische Chance guten Gewissens an uns vorbeigehen lassen? Dabei habe ich den mittel- und osteuropäischen Ländern immer wieder gesagt: Ihr steht völlig unter dem Bann von Big-Bang, von der Öffnung zur Marktwirtschaft usw. Aber gebt Acht! Ich habe den berühmten polnischen Premierminister und viele andere gewarnt: Passt auf! Markt ist nicht gleich Demokratie. Der Markt ist nützlich, doch die Demokratie ist etwas Anderes. Doch das haben sie nicht verstanden. Sie waren schlichtweg fasziniert von den Vereinigten Staaten und 
der NATO. Das hat Europa nicht in den Griff bekommen, aber trotzdem seine Arme weit geöffnet und sich großzügig wie eh und je gezeigt.

von Oppeln: Aber das heißt, dass sich mit der Osterweiterung wie auch mit dem Binnenmarkt und der Währungsunion die Bedingungen für die Stärkung der sozialen Dimension der Europäischen Union verändert haben. Genügt es heute also, zur Verstärkung der sozialen Dimension, die uns allen so am Herzen liegt, die Beitrittsländer zu unterstützen?

Delors: Nein, es reicht nicht aus, den neuen Beitrittsländern zu helfen. Ich nehme nichts von dem zurück, was ich bisher gesagt habe. Der soziale Dialog ist derzeit nicht das, was er sein sollte. Das sage ich nicht, weil ich ein Gewerkschaftler bin, sondern weil ich immer Respekt vor den Organisationen und ihrer historischen Bedeutung habe. Dies sowohl hinsichtlich der materiellen als auch der ideellen Interessen der Arbeitnehmer. Für mich ist das etwas Unverrückbares, ein Grundprinzip. Darüber hinaus beginnt das Soziale mit der Wirtschaft. Und daher rührt das berühmte mysteriöse Unverständnis zwischen Deutschland und Frankreich. Als mich der Europäische Rat auf Veranlassung von Helmut Kohl - dessen Verdienste für die deutsche Wiedervereinigung und Europa man gar nicht genug loben kann - aufgefordert hatte, einen Bericht über die Wirtschafts- und Währungsunion zu verfassen, haben wir mehr als die Hälfte des Berichts der Kooperation im Bereich der Wirtschaft gewidmet. Und was ist davon übrig geblieben? Nichts! Oder zumindest nicht viel. Und warum? Weil die Franzosen sofort sagten, dass Europa eine „Gouvernement économique“ (Wirtschaftsregierung) braucht. Die Deutschen verstehen das nicht. Und ich verstehe, warum die Deutschen das nicht verstehen. Denn für sie muss die Zentralbank in der liberalen Ordnung der sozialen Marktwirtschaft unabhängig sein. Umgekehrt wollen die Deutschen nicht akzeptieren, dass die Finanzminister bei ihren Zusammenkünften versuchen, ihre nationale Wirtschaftspolitik zu diskutieren, um sie bestmöglich aufeinander abzustimmen und die Vorteile zu maximieren. Seitdem ich nicht mehr in der Kommission bin, ist das nie wieder geschehen. Es wird also nicht mehr darüber diskutiert. Die Finanzminister reden nur noch über Haushaltsdefizite. Dabei ist das Budget nur ein Teil der Wirtschaftspolitik, ein Teil der Makroökonomie. Die Franzosen haben also immer wieder, auch während der Wahlkampagne, eine Wirtschaftsregierung gefordert. Die Deutschen - und da kann ich sie verstehen - mögen diesen Begriff nicht, und heute gibt es niemanden, der fähig wäre, ihnen begreiflich zu machen, dass man sich auf ein Modell einigen kann, das lediglich mehr Kooperation beinhaltet.

von Oppeln: Vielleicht ist es ja nur ein Problem der Formulierung? Vielleicht verstehen die Deutschen den Begriff Wirtschaftsregierung nicht?

Delors: Ja, sicherlich. Seit ich die Kommission verlassen habe, habe ich dieses Wort nie wieder in den Mund genommen, denn ich weiß, dass es dem Dialog nicht förderlich ist. Als ich meinen Weg durch Europa machte, musste ich Begriffe finden, die den Dialog, die Diskussion ermöglichten. Wenn auch nur ein einziger der Meinung ist, etwas sei undenkbar, untragbar und verstoße gegen seine Prinzipien, kommen wir zu keiner Lösung. Ich werde Ihnen noch ein Beispiel nennen. In den Jahren 1992 und 1993 ging es Europa nicht gut, denn Deutschland musste seine Wiedervereinigung mit drei bis vier Prozent seines 
Bruttosozialprodukts in einer starken Währung bezahlen. Und innerhalb des europäischen Währungssystems gab es Währungen mit einem starken Abwärtstrend - wie das britische Pfund Sterling oder die italienische Lira. Wenn Sie so wollen, waren also die positiven Auswirkungen des Binnenmarkts verschwunden. Daraufhin habe ich einen Bericht, ein so genanntes Weißbuch über Wettbewerb, Wachstum und Beschäftigung verfasst. Mit den deutschen Freunden hatte ich einige Schwierigkeiten. Ich musste dem Kanzler erklären, dass das, was man ihm erzählte, nicht stimmte. Schließlich wurde dieser Bericht vom Europarat angenommen, aber nie in die Tat umgesetzt. Warum nicht? Weil es vornehmlich um die Kooperation ging. Es ging nicht darum, der Union mehr Macht, mehr Kompetenzen zu erteilen, sondern es handelte sich um die Frage der Kooperation.

von Oppeln: ... und um die Frage der europaweiten Infrastruktur, der transeuropäischen Netze....

Delors: Ja die Infrastrukturen... Das hat also nicht funktioniert. Die Finanzminister traten zusammen und diskutierten endlos. Man könnte alles in einem Satz zusammenfassen: Die Außenminister sind europäischer eingestellt als die Finanzminister. Das ist meine Erfahrung. Die Außenminister finden immer einen Weg, ein Einvernehmen herzustellen. Aber die Finanzminister, was bringen sie gemeinsam zustande? Schauen Sie sich nur Herrn Juncker an. Man hörte nicht auf ihn, aber das war nicht sein Fehler. Die Zusammenarbeit fehlt eindeutig beim Aufbau Europas. Das gilt bis heute.

Das wird sich an der europäischen Wirtschafts- und Währungsunion zeigen, die schweren Zeiten entgegengeht, man sieht es auch im Bereich der Energiepolitik, bei der jeder seine eigene Strategie gegenüber Putin und Medvedev verfolgt. Uns stehen noch viele Probleme bevor, weil wir uns nicht auf ein Minimum an Kooperation einigen konnten und weil wir nicht aufrichtig und offen miteinander umgehen. Und das nach 50 Jahren Europa!

von Oppeln: Das heißt, dass wir es heute mit einem Trend zur Renationalisierung der europäischen Politik zu tun haben?

Delors: Ja, ein Rückzug auf sich selbst, weil die Bürger dies einfordern. Sie sind von der Globalisierung völlig überfordert, finden sich zwischen der lokalen und der globalen Welt nicht mehr zurecht. So suchen sie Sicherheiten, Leitbilder und Rückhalt. Demokratische Regierungen gehen unmittelbar darauf ein. Hinzu kommt, dass man es sich manchmal auch zu leicht macht. Die Bereitschaft zur europäischen Zusammenarbeit ist weniger stark ausgeprägt als in vergangenen Zeiten. Der Beweis - das wird auch Angela Merkel nicht gefallen: Im Kampf gegen die aktuelle Finanz- und Wirtschaftskrise gibt es viele Programme, aber kein gemeinsames europäisches Programm. Das ist besser als nichts. Wenn es brennt und die Feuerwehrleute von überall her das Feuer löschen, ist das nicht schlecht, aber es genügt nicht. Das ist die aktuelle Situation.

von Oppeln: Die viel diskutierte Frage der Wirtschaftsregierung bzw. der wirtschaftlichen Kooperation ist ein Problem, dass insbesondere von Deutschen und Franzosen 
diskutiert wird. Aber ist die Lösung dieser Frage nicht noch schwieriger, wenn man die neuen Mitgliedsstaaten einbezieht?

Delors: Nein, der große Irrtum innerhalb der Wirtschaftsunion besteht darin, an die Wirtschafts- und Währungsunion und die Union der 27 dieselben Kriterien anzulegen. Das erkennt man schon daran, dass die Minister der Wirtschafts- und Währungsunion am Vorabend tagen und die Kommission dieselben Prinzipien anwendet. Dabei ist das doch nicht dasselbe! Die Wirtschafts- und Währungsunion ist vergleichbar mit Schengen, um ein anderes Beispiel für verstärkte Zusammenarbeit und Differenzierung zu nennen. Das verpflichtet uns zu viel wichtigeren Dingen untereinander. Und es sind bestimmt nicht die neuen Mitgliedsstaaten, die uns davon abhalten. Bisher gehören nur Slovenien und die Slowakei der Eurozone an...

Es sind bestimmt nicht diese Länder, die für uns ein Hindernis darstellen. Das ist lediglich ein Problem deutsch-französischer Verständigung. Die Fehler liegen auf beiden Seiten, die anderen Länder ergreifen Partei. Die Holländer stehen eher auf deutscher Seite und sind recht unflexibel, die Belgier sind etwas offener, ebenso wie die Luxemburger... So sieht es aus. Dieses Problem ist nicht gelöst. Man hat den Eindruck, dass beide, die französische wie die deutsche Seite, die Dinge noch erschweren, um eine Verständigung zu verhindern. Dabei ist eine Einigung über eine Koordinierung der Wirtschaftspolitik statt einer Politisierung der Wirtschaftsunion durchaus möglich. Die Koordinierung ist möglich und notwendig. Diesen Vorschlag haben wir schon 1989 eingebracht. Doch nichts ist geschehen. Ich habe immer gesagt: Der Euro schützt uns, sogar vor unseren Dummheiten, doch er stimuliert nicht. Doch genau das müsste er tun. Europa braucht stets eine Keimzelle für Optimismus und Fortschritt. Natürlich schließen sich nicht alle Länder auf Anhieb an, doch früher oder später kommen sie dazu.

Blomert: Ist eine europäische Wirtschaftspolitik nur innerhalb der Euro-Zone oder auch mit Großbritannien möglich?

Delors: Nein, wir beginnen mit der Euro-Zone. Auf die Fortschritte, die wir dabei noch machen müssen, bin ich schon eingegangen. Großbritannien hat bis heute nie den echten Willen bekundet, soweit zu gehen. Das ist eine Tatsache. Tony Blair hat uns Geschichten vom Weihnachtsmann erzählt. Er hat ein Großbritannien hinterlassen, das Europa gegenüber noch feindlicher eingestellt ist als bei seinem Amtsantritt. Man muss die Dinge sagen wie sie sind. Dazu brauchen Sie sich nur die „soft réglementation“ im Bankenund Versicherungssektor anzuschauen, oder auch die Aufschreie der City oder sogar des Finanzministers Alistair Darling anzuhören. Wenn wir uns an England orientiert hätten, wäre Europa heute nicht dort, wo es ist. Es wäre vielmehr auf dem Rückzug. Wir haben den Beitritt der Engländer akzeptiert. Georges Pompidou und Willy Brandt trafen seinerzeit diese historische Entscheidung. Es gab viele gute Gründe für dieses Vorgehen, aber wir wollen uns nicht weiter mit ihnen aufhalten. Entweder sie folgen uns oder eben nicht. In der heutigen Welt mit Großmächten wie Indien, China, Brasilien usw. wird Europa im Jahr 2030 nur noch 3\% der Weltbevölkerung stellen. Was tun wir dann? Wie wollen wir weiter bestehen? Es geht um Überleben oder Niedergang. In Deutschland gibt es man- 
che, die meinen, das sei leeres Geschwätz, und Deutschland würde schon allein zurechtkommen. Nein! Denn obwohl Deutschland die stärkste Wirtschaftsnation ist, wird es das nicht schaffen. Die Deutschen müssen jedenfalls unbedingt einsehen, dass Europa auch Deutschlands Zukunft ist.

von Oppeln: Das vergessen die Deutschen heute manchmal...

Delors: Ja, die jüngere, nicht die ältere Generation. Meine über 60-jährigen deutschen Freunde erinnern sich sehr wohl noch daran. Wir wollen nicht unsensibel sein, aber es geht ja nicht nur darum, was vor 70 Jahren geschehen ist, sondern einfach darum, die Welt zu sehen, wie sie ist. Werden wir Europäer es schaffen, mit unseren Werten zu bestehen? Werden wir unseren Kindern ein Erbe hinterlassen, dem der Rest der Welt Respekt entgegenbringt? Genau darum geht es: Überleben oder Niedergang. Ich bedaure es sehr, dass viele Deutsche, sogar Regierungsbeamte, dafür kein Verständnis aufbringen!

Blomert: Wie schätzen Sie die aktuelle Finanz- und Wirtschaftskrise ein? Wenn sie weiter anhält, könnte sie dann nicht eine Chance sein, dieses Kontinentalmodell wieder herzustellen?

Delors: Nein, das Verdienst der französischen Ratspräsidentschaft unter Nicolas Sarkozy bestand darin, eine Tür aufgestoßen zu haben. Doch jetzt brauchen wir nicht nur einen Feuerwehrmann, sondern einen Architekten. Darin liegt das Problem ... einen Architekten Europas, für ein Europa, das ein weltweit wirkendes Beispiel für „soft regulation“ geben könnte. Das ist unsere Pflicht. Im Augenblick sind wir gute Feuerwehrleute und Herr Sarkozy hat getan, was er getan hat. Frau Merkel hatte auch ihre Vorstellungen. Doch jetzt geht es um die weitergehende Konstruktion Europas. Zuallererst muss Europa begeistern, die Menschen müssen von der guten Organisation überzeugt sein. Das bleibt noch zu beweisen.

von Oppeln: So wirken die Regierungen als Feuerwehrleute im jeweiligen nationalen Rahmen. Stattdessen sollte man, um der Konkurrenz der neuen Wirtschaftsmächte wie Indien, China, Russland und Südamerika standhalten zu können, Ihren Worten zufolge den Aufbau betreiben, doch in welche Richtung? Was genau muss man aufbauen? In erster Linie die wirtschaftliche Zusammenarbeit oder denken Sie noch an andere Projekte für die Zukunft dieses Aufbaus?

Delors: Seit meinem Amtseintritt waren die Föderalisten von mir stets enttäuscht. Warum? Weil ich niemals davon ausgegangen bin, dass es die Nationen nicht mehr geben würde. Daher habe ich versucht, ein System zu entwickeln, das ich „Föderation der Nationalstaaten" genannt habe und in dem die Nationen weiterhin bestehen bleiben würden. Doch diese Nationalstaaten sollten sich ihrer Grenzen bewusst sein. Wir sind schon ausführlich auf die wirtschaftliche Zusammenarbeit eingegangen, doch nehmen wir beispielsweise die Haltung der Mitgliedsstaaten gegenüber Russland, dem großen Energielieferanten. Da verhandelt jeder für sich, und Medvedev und Putin reiben sich die 
Hände. Soll das so weitergehen? Wir reden einerseits von gemeinsamer Außenpolitik, ernennen eine hohe Vertreterin für die Außen- und Sicherheitspolitik und haben nicht einmal eine gemeinsame Position über die Energiebeschaffung aus Russland. Diese Kluft muss überbrückt werden. Aus diesem Grunde ist auch eine Ausweitung der Kooperation erforderlich ... Ich verlange keine gemeinsame Außenpolitik. Das ist in der derzeitigen Situation ohnehin unmöglich. Aber wir sollten uns wenigstens als Europa würdig erweisen und unsere Interessen gegenüber Russland verteidigen! Ich würde gern mal in einem Beichtstuhl Angela Merkel, Nicolas Sarkozy, Silvio Berlusconi, Jose Luis Rodriguez Zapatero und Gordon Brown befragen. Da gäbe es so einige Überraschungen, wenn sie ehrlich sagen würden, was sie denken! Denn was sie wirklich denken, geht nicht in Richtung einer gemeinsamen europäischen Aktion. Jeder hat seine eigene Interessensphäre, seine eigene Vorstellung, seinen eigenen Platz in der Welt. Und nach mir die Sintflut. Und die Sintflut wird kommen! In 50 Jahren existieren wir nicht mehr. Dann gibt es nur noch einen G2, vielleicht einen G3, ohne uns.

von Oppeln: Das größte Problem liegt also im Mangel an Zusammenarbeit und Koordination?

Delors: Ja, und gleichzeitig fehlt eine Vision der Zukunft! Alles, was wir unserer Geschichte verdanken und was die Verfassung nicht aufnehmen wollte, nämlich unsere jüdisch-christliche Kultur, die Reformation, die Aufklärung, der muslimische Einfluss, die griechische Demokratie und Philosophie, das römische Recht, all das sind unsere Wurzeln, all das macht Europa aus..... Wer keine Erinnerung, kein Gedächtnis hat, hat auch keine Vision. Heute ist das alles in Vergessenheit geraten, weil jeder nur seine eigenen Interessen verfolgt, das ist kurzfristiges Denken. Es gibt keine einzige Regierung, die in Europa langfristig denkt.

von Oppeln: Aber es sind diese Werte, die die europäische Identität ausmachen?

Delors: Ja, es sind vor allem die Werte, da haben Sie Recht. Das Gleichgewicht zwischen Gesellschaft und Individuum, die Freiheit, die Demokratie, der Frieden, die Achtung vor dem Anderen, die Völkerverständigung. Nur wir haben das hervorgebracht. Stellen Sie sich vor, welche Bedeutung der Appell von Robert Schuman im Jahr 1950 hatte, und wie er von Adenauer und den anderen aufgenommen wurde. Das ist einfach großartig. Eines Tages habe ich zu einem Papst gesagt: Wissen Sie, Sie halten Europa immer für materialistisch. Dabei ist die größte spirituelle Triebfeder unserer Zeit doch genau das: Das Verzeihen und das Versprechen. Das Verzeihen, das nicht gleichbedeutend mit Vergessen ist und das Versprechen, dass wir alle zusammenleben werden. Das ist grandios. Seit 60 Jahren gab es sogar in spiritueller Hinsicht keine wichtigere Geste als diese. Doch wer spricht heute noch darüber?

von Oppeln: Wir haben kurz über die aktuelle Krise der Europäischen Union gesprochen. Es gibt dafür vermehrt Anzeichen. Eines dieser Anzeichen haben wir bereits erwähnt, nämlich eine Art von Renationalisierung, eine Rückbesinnung auf sich selbst. Ein weite- 
res Zeichen für die Krise ist auch die kritische Einstellung der Bevölkerung, die fehlende Identifikation mit der Europäischen Union. Will man die Bevölkerung mit der Europäischen Union wieder versöhnen, genügt es da, ihre Werte zu erklären oder muss nicht mehr geschehen?

Delors: Das ist eine Frage des gesunden Menschenverstands, nicht mal eine Frage der Politikwissenschaft, denn wir sind kein Staat wie die Vereinigten Staaten. Sie brauchen sich nur an die kritischen Stimmen bei der Nominierung von Herrn Van Rompuy zu erinnern. Man brauchte einen Präsidenten der Vereinigten Staaten, aber wir sind nicht die Vereinigten Staaten von Amerika, sondern wir sind ein Gebilde unterschiedlicher Völker und haben mehrere Verantwortliche, die diese Aufgabe in kollegialer Weise durchführen. Ich weiß nicht, wie die Presse bei Ihnen darauf reagiert hat, aber in Frankreich wurde die Ernennung eines Vermittlers als Katastrophe heraufbeschworen. Und wenn wir von Europa sprechen - ob es Herrn Kissinger nun passt oder nicht - dann gibt es da den Kommissionspräsidenten, den ständigen Präsidenten des Rates, die rotierende Präsidentschaft, die ja fortgesetzt wird, und den hohen Vertreter. Wir haben einen Vermittler, einen Chairman und keinen Präsidenten der Exekutive ernannt. Ich weiß nicht, wie es in Deutschland zugeht, aber bei uns verkündet ein Präsident bei seiner Rückkehr vom Treffen des Europäischen Rates: „Ich habe gewonnen.“ Acht Tage später sagt er: „Wissen Sie, Europa ist ganz fabelhaft, eine große Familie." Tatsächlich? Eine Familie, in der ein Bruder einen verbitterten Kampf führt, um gegen die anderen zu gewinnen? Aber die eigentliche pädagogische Arbeit an Europa kommt doch den nationalen Regierungen zu. Was soll Barroso tun, um die öffentliche Meinung zu Europa zu verändern? Das ist doch eher eine Aufgabe der nationalen Regierungen. Aus dieser Perspektive muss man die deutsche Ratspräsidentschaft loben, denn sie ist nicht triumphierend aufgetreten. Sie hat versucht, einiges zu verändern und ist dabei sehr professionell vorgegangen. In den anderen Ländern hingegen hieß es: Wenn unser Präsident, unser Premierminister nicht dabei gewesen wäre, was wäre dann aus uns geworden? Das ist lächerlich. Wenn wir eine Familie werden wollen, dann dürfen wir nicht sagen, dass wir gegen die anderen gewonnen haben. Wir dürfen auch nicht jedes Mal, wenn uns etwas nicht gefällt, die Schuld auf Brüssel schieben! Nein, so kann das nicht funktionieren. Man kann dem Kommissionspräsidenten nicht die Rolle der Regierungen aufbürden.

\section{Blomert: Und die Rolle von Frau Ashton? Ist das der englische Pudel?}

Delors: Das ist in erster Linie ein technisches Organisationsproblem. Die Europäische Kommission besteht aus mehreren Kommissaren, die für außenpolitische Belange zuständig sind. Wird man ihnen nun die Arbeit wegnehmen? Wird die Diskussion kollegial bleiben? Das ist die Frage. Und dann ist da noch der europäische diplomatische Dienst. Wie wird er konzipiert? Denn die Traditionen eines „Foreign Office“ sind nicht dieselben wie beim Quai d'Orsay oder bei Ihnen im „Auswärtigem Amt“. Was wird geschehen? Das ist alles noch ungeklärt. Ich hoffe nur, dass die für den Posten der hohen Vertreterin der EU für Außen- und Sicherheitspolitik bestellte Engländerin nicht alle Dinge an sich reißen wird, denn sie sollte ja auch die anderen berücksichtigen. Wirklich wichtig zu wissen ist, dass eine gemeinsame Außenpolitik heutzutage nicht möglich ist. Gemeinsame politische 
Aktionen in der Außenpolitik hingegen sind durchaus denkbar. Davon habe ich ja im Blick auf Russland schon gesprochen. Das ist möglich, aber mehr ist nicht drin. Wenn man höhere Ziele anvisiert, dann enttäuscht man die Bürger, denn man stellt ihnen Dinge in Aussicht, die man nicht realisieren kann.

von Oppeln: Wurde insgesamt mit dem Lissabon-Vertrag endlich eine Lösung für die schwierige Frage der institutionellen Reform der EU gefunden? Ist der Lissabon-Vertrag Ihrer Meinung nach zufriedenstellend?

Delors: Nein, wahrlich nicht, aber die Mitgliedsländer sind erschöpft. Sie werden sich erst in einigen Jahren wieder an eine Reform der Institutionen wagen. Ich habe aus Treue zu Europa für den Vertrag votiert, aber ich bin nicht davon überzeugt, weder von der ständigen Präsidentschaft, noch von dem Hohen Vertreter für Außen- und Sicherheitspolitik noch von den übrigen neuen Regelungen. Lediglich zwei Dinge sind interessant und korrespondieren überdies mit den Wünschen Ihrer nationalen Abgeordneten. Erstens die Tatsache, dass das europäische Parlament jetzt für das gesamte Budget verantwortlich ist, denn bisher unterschied man in obligatorische und nichtobligatorische Ausgaben. Und zweitens können die nationalen Parlamente das Subsidiaritätsprinzip geltend machen, was in etwa mit den Beschlüssen des Karlsruher Verfassungsgerichts übereinstimmt. Das sind positive Aspekte, die die Bürger beruhigen und zu einem besseren Verständnis der europäischen Organisation beitragen können. Doch was das Übrige angeht, wird viel Lärm um nichts gemacht.

von Oppeln: Brauchen wir denn weitere institutionelle Reformen?

Delors: Ja, aber alle sind müde und erschöpft. Sogar auf Englisch sagt man „fatigue“ (müde) für diesen Begriff. Wir, damit meine ich den von mir gegründeten Think Tank Notre Europe, werden einen Gesetzentwurf für eine Energiegemeinschaft einbringen, für die man einen der WWU ähnlichen Vertrag bräuchte. Denn mit der Energiepolitik kann das so nicht weitergehen. Wir verlieren viel zuviel Zeit und machen uns untereinander Konkurrenz. Dabei sollten wir doch gemeinsame Ziele verfolgen wie nachhaltige Entwicklung, die mit dem Klimaschutz, der Sicherheit der Energieversorgung und dem reibungslosen Funktionieren des Wettbewerbs bei uns verbunden ist. Und vielleicht kann auch ein europäischer Fonds zur Förderung von alternativen Energien bereitgestellt werden. Allerdings ist bei diesen alternativen Energien Vorsicht geboten. Denn all jene, die zur Herstellung von Agrotreibstoffen die Wälder roden, sind ein Angriff auf unsere Zukunft....

An dieser Stelle muss ich Kanzler Kohl meine Anerkennung zollen. Wir beide haben in Huston bei einem G7-Treffen bereits gewarnt: Passt auf die Wälder auf! Das haben wir schon vor geraumer Zeit, vor 20 Jahren, gesagt. Bewahrt den Baumbestand! Und trotzdem wird überall abgeholzt. In der Geoökonomie gibt es zwei wichtige Phänomene: Erstens die Eisschmelze, die als solche beunruhigend ist, aber den Kapitalismus dennoch dazu antreiben wird, neue Energietransportmittel ganz oben in der nördlichen Erdkugel zu finden. Und zweitens die Vernichtung der Wälder, die für das fundamentale Gleichgewicht des Planeten tatsächlich dramatisch ist. Doch hierzu muss Europa einige Projekte 
gemeinsam ins Auge fassen, die die Energie- und Klimapolitik stimulieren. Ein Finanzierungsfonds für alternative Energien und warum nicht eine gemeinsame Karbonsteuer und zu einem akzeptablen Preis? Denn gegenwärtig kostet die Tonne CO2 auf dem internationalen Markt nicht sehr viel. Das reicht nicht aus. In diesem Zusammenhang möchte ich noch einmal darauf hinweisen, dass unser Ziel ja nicht die Senkung der Erderwärmung ist, sondern wir lediglich verhindern wollen, dass sie um $2^{\circ}$ ansteigt. Das ist der Punkt, an dem wir uns derzeit befinden. Das Klima-Energie-Paket der Kommission ist gut, das muss man ihm lassen. Diese Kommission wurde häufig zu Unrecht kritisiert. Das ist ein gutes Paket. Es muss umgesetzt werden!

von Oppeln: Vielleicht könnte dieses Projekt etwas Neues für die europäische Politik bringen?

Delors: Es gibt zwei wichtige Projekte: Die Wiederherstellung des Gleichgewichts der Wirtschafts- und Währungsunion, um sie für die Zukunft zu stärken und das Klima-Energie-Paket mit der Möglichkeit einer europäischen Karbonsteuer und eines Fonds, der alternative Energien, aber nicht die Zerstörung von Wäldern finanziert.

von Oppeln: Eine Energie- und Energiebeschaffungsunion?

Delors: Ja, eine europäische Energie- und Klimaunion - wenn man das Klima dazu nehmen will - und eine echte Wirtschafts- und Währungsunion. Im Moment haben wir eine Währungsunion. Nach 10 Jahren können wir eigentlich recht zufrieden sein, aber ich bin immer noch kritisch. In diesen 10 Jahren hatten wir ein Wachstum von 2,1\% und haben 15 Millionen Arbeitsplätze innerhalb der EU geschaffen. Doch heute - wenn ich noch ein junger Mann von 40 Jahren wäre - würde ich mit meinem Pilgerstab zu Herrn Weber, dem Präsidenten der Bundesbank, gehen, damit er den Deutschen sagt, dass sie doch ein Minimum an zwischenstaatlicher Koordinierung der Wirtschaftspolitiken akzeptieren sollen....

Ich würde jedenfalls so handeln. Ich war immer dafür... Das Fundament all unserer Errungenschaften war doch stets ein großes Einverständnis mit Deutschland. Ich habe schon 2 Tage nach dem Berliner Mauerfall verkündet, dass die Ostdeutschen zu Europa gehören. Da haben manche vielleicht komisch geguckt! Ich habe jedenfalls mein Handeln immer darauf gegründet. Und heute träume ich davon, dass jemand diese Fackel in die Hand nimmt und den Deutschen sagt: Achtung, es geht euch zwar gut und ihr thront im Zentrum Europas! Aber gebt euch damit nicht zufrieden!

von Oppeln: Ja, aber derzeit sind es eher die Franzosen, die auf der Suche nach einer neuen Agenda für die deutsch-französische Zusammenarbeit in Europa sind.

Delors: Ja, ich habe den Eindruck, dass sich Ihre Regierung weniger dafür interessiert... Vielleicht haben wir nicht die richtigen Argumente. Deshalb wünsche ich mir so sehr, dass jemand mit seinem Pilgerstab die Deutschen überzeugen würde. Aus freundschaftlicher Verbundenheit, Respekt und Erkenntnis, aber so jemand fehlt. Es reicht nicht zu sagen, dass man einen gemeinsamen Minister zweier Regierungen aufstellen will. Wozu soll das dienen, wenn niemand daran glaubt? 
von Oppeln: Also dann sind es Ihrer Meinung nach eher die Deutschen, die eine fruchtbare Zusammenarbeit beider Länder in Europa erschweren?

Delors: Nein. Die Deutschen müssen nur überzeugt werden. Und dafür finden wir scheinbar nicht die richtigen Argumente. Wir müssen Überzeugungsarbeit leisten, vor allem bei den jüngeren Generationen der 30- bis 40-Jährigen, da steht uns noch einige Arbeit bevor. Denn angesichts der Globalisierung und der Tatsache, dass Deutschland die stärkste Wirtschaftsmacht Europas ist, verstehe ich das Verhalten der Deutschen. Aber ich glaube auch, dass den Deutschen nicht alle Gefahren bewusst sind und sie nicht auf alle Herausforderungen reagieren, die die Europäer in ihrer Gesamtheit betreffen und die im Grunde dafür sorgen, dass sich die Welt bewegt.

von Oppeln: Hat sich das Verhältnis umgekehrt? Früher waren es eher die Franzosen, die von den Deutschen überzeugt werden mussten, und heute sind es die Deutschen, die überzeugt werden müssen. Hat zwischen den beiden Ländern ein Rollentausch stattgefunden?

Delors: Die Deutschen müssen davon überzeugt werden, - nicht unbedingt von den Franzosen - dass Europa ihre Zukunft ist. Das ist alles. Doch es ist wahr, vor mittlerweile 20 Jahren musste ich meinen Pilgerstab in die Hand nehmen, um die Franzosen davon zu überzeugen, dass die brüderliche Union mit Deutschland unausweichlich ist. Und das habe ich getan. Es war nicht immer leicht für mich, denn ab und zu hat man mir meine angeblich übertriebene Deutschfreundlichkeit vorgehalten.

Blomert: Glauben Sie, dass ein Teil dieser europäischen Architektur auch eine europäische Armee umfassen muss?

Delors: Das halte ich für verfrüht. Meine Ziele sind bescheidener. Im Grunde gibt es drei Ziele, von denen ich bisher nur zwei genannt habe: die Wiederherstellung des Gleichgewichts der Wirtschafts- und Währungsunion und eine Energie- und Klimagemeinschaft. Und drittens würde ich sagen, eine Vertiefung unserer Beziehungen mit den Vereinigten Staaten, die über die Zukunft des atlantischen Bündnisses bestimmt. Wir müssen unsere Beziehungen mit den USA ganz unsentimental neu überdenken und uns dabei nicht von Primärreflexen wie ,ich bin dafür“ oder ,ich bin dagegen“ "leiten lassen. Diese drei Punkte müssten meines Erachtens auf der Agenda der deutsch-französischen Treffen stehen. Ich behaupte nicht, dass Deutschland und Frankreich allein Europa ausmachen. Auch wenn Deutschland und Frankreich jahrzehntelang in Europa eine führende Rolle innehatten, habe ich stets auf den Einfluss der anderen Länder verwiesen: das Spanien von Gonzalès, das Italien von Andreotti, das Belgien von Martens und Dehaene, die Niederlande von Herrn Lubbers und das Luxemburg von Herrn Santer. Es gibt also nicht nur sie, doch wenn man sich auf die vordringliche Angst konzentriert, die Deutschen und Franzosen könnten sich nicht verstehen, würde ich mit diesen drei Vorschlägen antworten.

von Oppeln: Kommen wir noch auf ein anderes Thema, nämlich auf das Problem der EUErweiterung. Mit dem Inkrafttreten des Lissabon-Vertrags fällt ja ein stichhaltiges Argu- 
ment gegen den Beitritt neuer Länder wie der Türkei weg. Welchen Standpunkt beziehen Sie zu dieser Frage?

Delors: Man muss zwei Dinge unterscheiden. Zum einen die Unstimmigkeiten zwischen Deutschland und Frankreich bei der Jugoslawien-Tragödie seit 1991. Das war für mich sehr schmerzhaft, doch ich konnte nichts dagegen ausrichten. Nach meinem Dafürhalten ist in den Balkanländern und in Ex-Jugoslawien die Botschaft Robert Schumans vonnöten: das Verzeihen und das Versprechen. Wir haben eine historische Pflicht gegenüber diesen Völkern. Doch sind wir dieser Pflicht bisher nachgekommen? Da bin ich mir nicht so sicher. Alle diese Länder gehören zu Europa. Wenn das ursprüngliche Ziel der Gründerväter Europas der Friede war, dann bleibt uns noch viel zu tun. Die große Feindschaft und Verbitterung, die nach dem Ende des 2. Weltkriegs zwischen den Deutschen einerseits und den Franzosen, Niederländern und Belgiern andererseits herrschte, haben wir überwunden. Doch in Ex-Jugoslawien ist uns das nicht gelungen. Das müssen wir schaffen! Doch dazu müssen wir uns etwas mehr anstrengen und konsequenter sein als bisher... Man muss eine geopolitische Vision entwickeln - das ist unsere Pflicht...

Mit der Türkei verhält sich das anders. Bei der Türkei habe ich nie das Wort „Nein“ ausgesprochen. Ich war Verhandlungen gegenüber stets offen. Ich kritisiere all jene, die sich für ein endgültiges ,Nein“ entschieden haben. Warum? Weil wir es in der Welt mit einer ideologischen Schlacht mit fundamentalistischem Gedankengut zu tun haben, das die Existenz des Anderen negiert und alles beherrschen will. Wenn wir die Türkei definitiv ausschließen, dann gießen wir Wasser auf deren Mühlen. Dazu kommt, dass wieder Andere sagen, natürlich, das ist dieses christliche Europa. Daher der Hass. Ich glaube, dass das unumstößliche Nein uns beim Kampf um die Werte und die Ideologie sehr geschadet hat. Ich stehe da nicht allein. Michel Rocard vertritt beispielsweise in Frankreich denselben Standpunkt wie ich. Aber ich bedaure, dass einige Nein gesagt haben. Die Meinungen sind geteilt. Ich könnte auch politisch argumentieren: Die Türkei spielt nicht zuletzt gegenüber dem Iran, Israel und Palästina eine wichtige und häufig unterschätzte Rolle. Wir müssen achtsam sein. Vielleicht ist die Türkei auch nicht fähig, die erforderlichen Bedingungen zu erfüllen, aber diese kategorische Ablehnung hat uns großen Schaden zugefügt. Das lässt sich nicht leugnen.

von Oppeln: Der Schaden ist vielleicht schon angerichtet.

Delors: Ja, aber man kann versuchen, ihn wieder gutzumachen.

von Oppeln: In dem Sinne, dass die Türkei in die EU integriert wird?

Delors: Nein, wir müssen mit ihr verhandeln und ihr für ihre Außenpolitik die ihr gebührende Anerkennung zollen. Das ist alles. Ich verfolge diese Entwicklung genau, obgleich mir natürlich nicht dieselben Informationen wie unseren diplomatischen Diensten vorliegen. Doch jedes Mal, wenn mir jemand in meinem Umfeld erzählt, dass die Türken dieses oder jenes getan hätten, ist das für mich „Realpolitik“. So argumentiere ich nicht. Für mich zählt vor allem der Kampf gegen den Fundamentalismus, gegen die Ideologie 
der Ablehnung des Anderen. Was hat denn in Europa zu blutigen Auseinandersetzungen geführt? Eben diese Ausgrenzung, der Hass auf das Andersartige. Und das wollen wir tatsächlich akzeptieren und damit dem Rest der Welt als Vorbild dienen? Das kann doch wohl nicht sein!

von Oppeln: Glauben Sie, dass sich die Türkei mit endlosen Verhandlungen zufrieden geben würde?

Delors: Ja, wenn es eine offene, ehrliche Sprache ist, wird sie sich Zeit nehmen, um besser abschätzen zu können, wozu sie sich verpflichtet. Denn es gibt viele interne Probleme in der Türkei, und es werden viele Kämpfe ausgetragen zwischen der gemäßigten islamistischen Partei, ich würde sagen einer Art christliche Demokratie nach muslimischem Muster, und den Nationalisten, den Schülern der Laizität. Hinzu kommt das Kurdenproblem, das nicht nur ein türkisches Problem ist. Doch sie haben im Moment den Eindruck, dass man ihnen tatsächlich den Beitritt verwehrt hat. Es wird zwar weiter verhandelt, aber mehr aus Prinzip. Alle werden immer sturer. Und niemand geht als Gewinner hervor.

von Oppeln: Die Türken denken bereits über Alternativen nach.

Delors: Ja, und das ist ein Spiel ohne Gewinner. Ich kann dieses kategorische ,Nein“ nicht verstehen. Ebenso wenig übrigens wie das bedingungslose ,Ja‘.

von Oppeln: Und die anderen Länder wie z. B. die Ukraine?

Delors: Das ist wesentlich komplizierter, denn man muss sich zunächst mit Russland einigen, und dieses Land ist nicht ganz unproblematisch. Ich denke ... ich kann nicht eindeutig Ja oder Nein sagen. Es ist schwierig, wenn ich mir alle umliegenden Länder anschaue. Und dann bereitet mir unser Verhältnis zu Russland Sorge. Ich finde, wir treten nicht entschieden genug auf, aber das ist meine persönliche Meinung.

Blomert: Würde das wie eine Offensive gegen Russland aussehen?

Delors: Ja, ich bekomme sehr wohl mit, dass Russland alte Zeiten herbeisehnt, ähnlich wie Frankreich, das während der Postkolonialzeit seinen Kolonien nachtrauerte. Russland hat ein nostalgisches Verhältnis zur Sowjetunion.

Blomert: Es bleibt ihnen nichts Anderes übrig.

Delors: Das ist klar. Überdies sind sie auch machiavellistisch, insbesondere in punkto Energie. Sie spalten uns. Aber man kann ihre Angst verstehen und mit ihnen verhandeln. Doch sind unsere Beziehungen mit Russland nach wie vor ungeklärt. Würde ich das Problem in seiner ganzen Komplexität behandeln, würde niemand meine Gedanken aufgreifen. Doch wenn man es nur auf die Frage der Energie eingrenzte, könnte uns das weiterbringen. 
von Oppeln: Generell möchte ich hinsichtlich der Erweiterung die Frage stellen, ob man die Grenzen Europas definieren muss oder kann?

Delors: Nein, die Globalisierung verwehrt uns dies. Wir müssen vernünftiger und in gewisser Weise vorsichtiger sein. Ich hatte von den europäischen Werten gesprochen. In der Bescheidenheit meiner Person glaube ich an diese Werte, die übrigens über die derzeitigen Grenzen Europas hinausreichen. Es gab in den Ländern, die heute Beitrittskandidaten sind, Literaten und Künstler usw., die europäisch gedacht haben. Doch das, was vor allem den Franzosen fehlt, ist die Einfühlsamkeit für diese Länder. In unserer Organisation „Notre Europe“ haben wir diesbezüglich große Anstrengungen unternommen. Wir haben ein Buch herausgebracht, das Gespräche mit Vertretern dieser Länder enthält. Wir haben versucht, die Bedenken und Wünsche der Menschen zu verstehen. In der Tagespolitik kann man so etwas nicht tun. Aber im Umfeld der Politik muss das Verständnis gefördert werden, denn die Integration Europas gründet auf den Werten und auf der Verständigung zwischen den Völkern.

von Oppeln: Sie stehen der Erweiterung recht offen gegenüber, aber steht diese nicht im Widerspruch zu all den von ihnen genannten Projekten, die auf eine engere wirtschaftliche Zusammenarbeit und Koordination abzielen?

Delors: Das Gegenstück wäre die Entwicklung der verstärkten Zusammenarbeit. Europa kann sich nicht ohne jede Differenzierung gleichzeitig erweitern und vertiefen. Wäre eine Einstimmigkeit erforderlich gewesen, hätte es Schengen und den Euro nie gegeben. Wir müssen auf diesem Weg voranschreiten, im Respekt der anderen. Aber wir brauchen diesen Antriebsmotor. Ich meine verstanden zu haben, dass Frau Merkel die verstärkte Zusammenarbeit ablehnt. Ich halte dies für einen Irrtum. Wir brauchen einerseits eine breite Vision von Europa in der Welt von morgen und andererseits die Möglichkeit, der Bewegung neue Impulse zu geben. Dies kann nur von einer kleineren Gruppe von Ländern ausgehen, die den anderen gegenüber offen sind. Wenn wir das nicht akzeptieren, wird Europa ein lockeres Bündnis. Wenn wir immerhin einen Binnenmarkt behielten, wäre es schon nicht schlecht, selbst ohne Außenpolitik oder gar gemeinsame Handelspolitik. So sieht der Niedergang aus.

...Ohne Antriebsmotor, der die Lebensgeister wachrüttelt, schlummern die Völker ein, und die Regierungen können ihnen Geschichten, Märchen vom Weihnachtsmann, erzählen. Und dann kommen wir nicht voran. Wir brauchen ständig einen Antriebsmotor. Gegenwärtig ist das Klima unsere treibende Kraft. Man kann sagen, was man will, aber die Umweltschützer bringen alles durcheinander und geben uns dadurch neue Impulse. Sie machen uns Angst. Sie haben ganz Recht. Europa muss Leute haben, die sich Sorgen machen, die sagen, dass das so nicht bleiben kann. In der Geschichte braucht man neue Initiativen. Dann werden sie von den Staaten, den demokratisch gewählten Abgeordneten ratifiziert, aber man braucht Antriebsfedern.

Blomert: Und man braucht Personen... 
Delors: Ja, die auch. Man braucht Menschen, die das alles verkörpern. Leider ist das so, aber man braucht sie.

von Oppeln: Ich möchte noch einmal kurz auf die Visionen zurückkommen. Sie gehören ja einer Generation an, die Europa und die europäische Union mit aufgebaut hat.

Delors: Ich nicht, das war die Generation vor mir. Da bin ich bescheiden. Die Generation, die Europa aufgebaut hat, war meine Vorgängergeneration.

von Oppeln: Aber Sie haben doch wirklich maßgeblich zum Aufbau Europas beigetragen.

Delors: Nicht ich, ich gehöre nicht zu den Gründervätern. Pech für mich, ich wäre gern 10 Jahre älter und hätte dies vollbracht.

von Oppeln: Ich für meinen Teil gehöre der Generation an, die mit dieser Europäischen Union aufgewachsen ist. Für mich gab es sie schon immer. Doch lassen Sie uns jetzt einen Blick in die Zukunft werfen. Kann man heute davon ausgehen, dass es die Europäische Union immer weiter geben wird, oder besteht die Gefahr, dass wir uns zu sicher fühlen?

Delors: Sie haben Recht. Nichts ist für ewig verbürgt. Es wird weiterhin Jahrestage geben, die Regierungschefs werden dieses oder jenes feiern, aber die Union verliert immer mehr an Dynamik. Das kann dazu führen, dass wir in der Welt nichts mehr zu sagen haben. Denn die Vereinigten Staaten sind präsent, China ist mächtig und Indien ... Sie brauchen sich nur die Diskussionen in der WTO mit Brasilien und Südafrika anzuschauen. 24 Länder haben gerade ein Abkommen getroffen und uns an der Nase herum geführt. 24 Entwicklungsländer haben sich auf einen freieren Handelsaustausch untereinander geeinigt. Die Welt verändert sich. Ich denke da an unsere Enkelkinder, nicht mal mehr an unsere Kinder. Ob sie wohl in einer Welt leben werden, die von den anderen praktisch kolonialisiert ist? Denken Sie nur an die Chinesen, die afrikanische Farmen aufkaufen und an die Hedge Fonds. Wie kann man heutzutage Staatschef einer französischen Regierung und Europäer sein und sich keine Sorgen um die Zukunft machen und keine schlaflosen Nächte mit der quälenden Frage verbringen: Ich bin an der Macht. Was kann ich nur tun, um diesen Niedergang zu verhindern? Das kann ich nicht begreifen. Natürlich haben Staatsoberhäupter das Problem der öffentlichen Meinung, die Medien werden immer einflussreicher, aber beschäftigen sie sich wirklich mit diesen Fragen? Ich jedenfalls denke in langfristigen Kategorien. Eines Tages werden wir zur Rechenschaft gezogen. Habe ich tatsächlich an meinen Grundwerten, an meinem Land und an Europa festgehalten?

von Oppeln: Was würden Sie Europa am dringlichsten empfehlen?

Delors: Das sagte ich ja schon: Die Wirtschafts- und Währungsunion, eine verstärkte Zusammenarbeit in Sachen Energie und Klima und die Klärung der Beziehungen mit den 
Vereinigten Staaten und Russland. Das sind brenzlige, dringende Punkte, die unbedingt auf der Agenda stehen müssen.

von Oppeln: Und was sind Ihre größten Befürchtungen? Der Niedergang Europas?

Delors: Der Niedergang Europas als Machtfaktor und der Werteverfall.

Blomert: Bis wann ungefähr? 10 Jahre? Eine Generation oder zwei Generationen?

Delors: Die Historiker lehren uns, dass ein Niedergang schnell oder langsam vonstatten gehen kann. Dafür gibt es genügend Beispiele. In totalitären Systemen tritt der Niedergang eher brüsk ein. Aber er kann auch schleichend sein. Die Leute merken es nicht sofort, sondern es wird ihnen erst hundert Jahre später bewusst, dass sie nicht mehr mit im Spiel sind. Das kommt vor. Die Europäer haben im Schnitt einen recht hohen Lebensstandard ... Dass es auch Arme gibt, weiß ich nur zu gut, das braucht mir keiner zu sagen. Wir müssen uns über die Gefahren, die uns und unseren Lebensstandard bedrohen, bewusst werden.

...Das ist ein Problem für Menschen, die denken wie ich und die Handlungsmöglichkeiten haben oder die entsprechende Autorität besitzen, d.h. den Pilgerstab in die Hand nehmen und das alles erklären, aber auch zuhören können. Es fehlt dieser Pilger, ... Ich hatte diese Rolle im zweiten Halbjahr 1984. Ich bin in alle Länder gereist, habe einen Haufen Dinge ausprobiert. Ich hatte ein Ohr für andere, auch für Frau Thatcher, mit der ich mich trotz unserer Differenzen auf menschlicher Ebene sehr gut verstanden habe. Wenn Frau Thatcher ein innenpolitisches Problem hatte, von dem auch Europa hätte betroffen sein können, bin ich nach London gereist und habe ihr zugehört. Es muss Leute geben, die einsatzbereit sind und im europäischen Sinne argumentieren.

von Oppeln: Doch wo sind heute die Kräfte, mit denen man in dieser Weise für Europa wirken kann?

Delors: Man muss innerhalb jeder Partei, jedes Arbeitgeberverbands, jeder Gewerkschaftsorganisation und jedes Vereins der Zivilgesellschaft arbeiten. An allen Ecken und Enden. Überall dort, wo es Leute gibt, die leichtfertig sind und nicht über die Zukunft nachdenken, die meinen, das ginge schon alles irgendwie. In jeder Organisation müssen Leute sein, die sich Sorgen um die Zukunft machen. Doch mitunter haben ihre Gründe zur Besorgnis weniger Belang als die, über die wir eben gesprochen haben.

von Oppeln: Wo bleibt beispielsweise das Engagement der Parteien? Es gibt vielfältige Vereine, die vielzitierte Zivilgesellschaft, aber ist das Engagement der Parteien und der Gewerkschaften ausreichend?

Delors: Sie brauchen Hilfe. Und natürlich den sozialen Dialog, der zu meiner Zeit noch gut funktionierte, denn er wurde von der technischen Assistenz der Kommission und meinen Mitarbeitern unterstützt. Nicht um ihnen vorzuschreiben, was sie tun sollten, sondern 
um sie zu unterstützen. Um ihnen den Eindruck und die Sicherheit zu geben, dass wir mit unseren Aktionen nicht eine soziale Klasse gegen eine andere ausspielen wollten. Nein, beim freien Warenaustausch und dem Binnenmarkt ging es nicht nur um Liberalisierung und Deregulierung, sondern das war auch eine Angelegenheit der gesamten Gesellschaft. Das muss immer wieder richtig gestellt und x-mal wiederholt werden.

von Oppeln: Noch eine letzte Frage: Haben Sie den Eindruck, dass die künftige EUKommission, zu deren Zusammensetzung bereits Entscheidungen vorliegen, in der Lage ist, diese Aufgabe zu bewältigen?

Delors: Ich habe Vertrauen in die Institution. Ich spreche niemals von Personen. Ich möchte, dass Sie dies hervorheben, denn Herr Barroso macht sich manchmal Sorgen. Nein, ich habe nie von Personen gesprochen. Ich spreche von Institutionen. Und im Sinne der institutionellen Konzeption habe ich auch verlangt, dass ein „Chairman“ und kein Exekutivpräsident à la Blair ernannt wird. Das habe ich Herrn Sarkozy wiederholte Male erklärt. Genau darum geht es. Daher habe ich Vertrauen in die Institution. Ich gehe von einer Konzeption der Gemeinschaft aus, nicht im Sinne des philosophischen Begriffs, sondern im Sinne des europäischen Gemeinschaftssystems, mit dem Parlament, dem Ministerrat und der Kommission. Jeder hat eine bestimmte Funktion. Und wenn jeder seine Aufgabe erfüllt, geht es mit Europa voran. Wenn diese Rollen hingegen vernachlässigt werden, macht Europa Rückschritte und wird zu einer intergouvernementalen und chaotischen Organisation. Doch ich habe Vertrauen in die Kommission.

von Oppeln/Blomert: Herr Delors, wir danken Ihnen für dieses Gespräch!

Nachbemerkung: Jacques Delors, den französischen Sozialisten und der christlich-sozialen Gewerkschaftsbewegung verbundener Ökonom und Politiker, hatte verschiedene Positionen in der französischen Politik, bevor er unter Präsident Mitterrand Finanz- und Wirtschaftsminister wurde. In dieser Funktion hat er 1983 unter dem Druck des neoliberalen Mainstreams die Wende von einer nachfrageorientierten Wirtschaftspolitik zu einer stabilitätsorientierten Politik durchgeführt.

Den Höhepunkt seiner politischen Karriere erreichte er als Präsident der Europäischen Kommission: In den zehn Jahren von Januar 1985 bis Dezember 1994 hat die Kommission den europäischen Integrationsprozess dynamisch vorangetrieben. Die Unterzeichnung der Einheitlichen Europäischen Akte, die Vollendung des Europäischen Binnenmarktes, der Abschluss des Maastrichter Vertrages zur Begründung der Europäischen Union und der Wirtschafts- und Währungsunion, die Einführung des sozialen Dialogs zwischen Gewerkschaften und Arbeitgebern auf europäischer Ebene sowie die erste Erweiterung der Union nach dem Fall des Eisernen Vorhangs von 12 auf 15 Mitgliedstaaten fallen in seine Amtszeit.

Seit dem Ende seiner Amtszeit als Präsident der Europäischen Kommission engagiert sich Jaques Delors weiterhin in der französischen und europäischen Politik. Er war Gründer und erster Präsident der in Paris angesiedelten europapolitischen Denkfabrik „Notre Europe“ und er erhielt vielfältige Ehrungen und Auszeichnungen, u. a. im Jahre 1992 den Karlspreis.

Das Gespräch mit Herrn Jacques Delors wurde am 4. Dezember 2009 in Paris von Dr. Reinhard Blomert und Dr. Sabine von Oppeln geführt.

Übersetzung aus dem Französischen von Angelika Kempf, Bearbeitung Dr. Sabine von Oppeln. 